\title{
Sparsity-Regularized Born Iterations for Electromagnetic Inverse Scattering
}

\author{
H. Bağc1 ${ }^{*(1)}$, R. Raich ${ }^{(2)}$, A. E. Hero ${ }^{(1)}$, and E. Michielssen ${ }^{(1)}$ \\ (1) Department of Electrical Engineering and Computer Science, \\ University of Michigan, Ann Arbor, MI 48109, USA \\ (2) School of Electrical Engineering and Computer Science, \\ Oregon State University, Corvallis, OR 97331, USA
}

\section{Introduction}

Electromagnetic inverse scattering [1] continues to be an active research area with applications ranging from environmental sensing to oil exploration and see-through-wall (STW) imaging. Among the many available techniques, the Born iterative method and its many descendants continue to be the most widely used [2]. The ill-posedness of Born iterations has often been alleviated via Tikhonov regularization [1], which promotes smoothness in the reconstruction. That said, in many applications including molecular and STW imaging [3], sparseness of the scatterers can be used to regularize the inverse scattering problem as well. Sparsity regularization was first proposed for linear inverse problems in [4] and then extended for image processing applications in [5-6]. In this paper, an inverse scattering technique that uses sparsity-regularized Born iterations is proposed. Application of the proposed technique to the reconstruction of a sparse twodimensional (2D) dielectric profile shows that it produces images that are sharper than those obtained using Tikhonov-regularized Born iterations.

\section{Formulation}

\section{A. Discretization of Electromagnetic Equations}

Consider a cylindrical ( $z$-invariant) scatterer $V$ that resides in a background medium with permittivity $\varepsilon_{0}$ and permeability $\mu_{0}$. Let $\varepsilon_{\mathrm{r}}(x, y)$ denote $V$ 's relative permittivity. A finite set of receivers $(R \mathrm{~s})$ and a line-source transmitter $(T)$ are located around $V$ (Fig. 1). The transverse magnetic (TM) field

$$
E_{z}^{\mathrm{inc}}(x, y)=-0.25 \omega \mu_{0} I_{\mathrm{s}} H_{0}^{(2)}\left(k_{0} \sqrt{\left(x-x_{\mathrm{T}}\right)+\left(y-y_{\mathrm{T}}\right)}\right),
$$

produced by the transmitter, which carries a current $I_{\mathrm{s}}$ and is located at $\left(x_{\mathrm{T}}, y_{\mathrm{T}}\right)$, induces volume polarization currents $J_{z}(x, y)$ in $V$. These currents satisfy the integral equation

$$
E_{z}(x, y)=E_{z}^{\mathrm{inc}}(x, y)-0.25 \omega \mu_{0} \iint_{V} J_{z}\left(x^{\prime}, y^{\prime}\right) H_{0}^{(2)}\left(k_{0} \sqrt{\left(x-x^{\prime}\right)+\left(y-y^{\prime}\right)}\right) d x^{\prime} d y^{\prime} .
$$

Here, the total field $E_{z}(x, y)$ satisfies

$$
J_{z}(x, y)=j \omega \varepsilon_{0} \delta \varepsilon(x, y) E_{z}(x, y),
$$

$\omega$ is the frequency, $k_{0}=\omega \sqrt{\varepsilon_{0} \mu_{0}}$ is the background medium's wave number, and $\delta \varepsilon(x, y)=\varepsilon_{\mathrm{r}}(x, y)-1$ is the scatterer's contrast. To discretize (2) and (3), $V$ is approximated by square cells, and $\delta \varepsilon(x, y)$ and $J_{z}(x, y)$ are approximated as 


$$
\delta \varepsilon(x, y)=\sum_{k=1}^{N} d_{k} p_{k}(x, y), \quad J_{z}(x, y)=\sum_{k=1}^{N} j_{k} p_{k}(x, y),
$$

where the basis function $p_{k}(x, y)$ is 1 for $(x, y) \in$ cell $k$, and zero elsewhere. Inserting (4) into (2), and evaluating the resulting equation at cell centers $\left(x_{k}, y_{k}\right), k=1, \ldots, N$, yields

$$
\mathbf{E}_{\mathrm{v}}^{\mathrm{scat}}=\mathbf{E}_{\mathrm{v}}-\mathbf{E}_{\mathrm{v}}^{\mathrm{inc}}=\mathbf{G}_{\mathrm{v}} \mathbf{J} .
$$

Here, $\mathbf{E}_{\mathrm{v}}^{\text {scat }}, \mathbf{E}_{\mathrm{v}}$ and $\mathbf{E}_{\mathrm{v}}^{\mathrm{inc}}$ are vectors of scattered, total, and incident field values evaluated at $\left(x_{m}, y_{m}\right), \quad m=1, \ldots, N ; \quad \mathbf{J}(k)=j_{k}, \quad k=1, \ldots, N$, is a vector of current coefficients (see (4)), and the entries of the matrix $\mathbf{G}_{\mathrm{v}}$ are [7]

$$
\mathbf{G}_{\mathrm{v}}(m, k)=\left\{\begin{array}{l}
-\omega \mu_{0} \pi a_{k} /\left(2 k_{0}\right) J_{1}\left(k_{0} a_{k}\right) H_{0}^{2}\left(k_{0} \sqrt{\left(x_{m}-x_{k}\right)+\left(y_{m}-y_{k}\right)}\right), m \neq k, \\
-\omega \mu_{0} \pi a_{k} /\left(2 k_{0}\right) H_{0}^{1}\left(k_{0} a_{k}\right)+j \omega \mu_{0} / k_{0}^{2}, m=k
\end{array},\right.
$$

where $a_{k}$ is the radius of the circle that has the same area as $k^{\text {th }}$ cell. Inserting (4) into (3) and evaluating the resulting equations at cell centers yields

$$
\mathbf{J}=j \omega \varepsilon_{0} \operatorname{diag}\{\mathbf{d}\} \mathbf{E}_{\mathrm{v}},
$$

where $\mathbf{d}(m)=d_{m}, m=1, \ldots, N$, is a vector of contrast coefficients (see (4)). Equation (2) also holds true at receiver locations $\left(x_{\mathrm{R}}^{m}, y_{\mathrm{R}}^{m}\right), m=1, \ldots, N_{\mathrm{R}}$; inserting (4) into (2) and evaluating the resulting equation at $\left(x_{\mathrm{R}}^{m}, y_{\mathrm{R}}^{m}\right), m=1, \ldots, N_{\mathrm{R}}$, yields

$$
\mathbf{E}_{\mathrm{R}}^{\text {scat }}=\mathbf{E}_{\mathrm{R}}-\mathbf{E}_{\mathrm{R}}^{\text {inc }}=\mathbf{G}_{\mathrm{R}} \mathbf{J} \text {. }
$$

Here, $\mathbf{E}_{\mathrm{R}}^{\text {scat }}, \mathbf{E}_{\mathrm{R}}$ and $\mathbf{E}_{\mathrm{R}}^{\text {inc }}$ are vectors of scattered, total, and incident field values evaluated at $\left(x_{\mathrm{R}}^{m}, y_{\mathrm{R}}^{m}\right), m=1, \ldots, N_{\mathrm{R}}$, and the entries of the matrix $\mathbf{G}_{\mathrm{R}}$ are

$$
\mathbf{G}_{\mathrm{R}}(m, k)=-\omega \mu_{0} \pi a_{k} /\left(2 k_{0}\right) J_{1}\left(k_{0} a_{k}\right) H_{0}^{2}\left(k_{0} \sqrt{\left(x_{\mathrm{R}}^{m}-x_{k}\right)+\left(y_{\mathrm{R}}^{m}-y_{k}\right)}\right) .
$$

\section{B. Sparsity Regularization}

Consider a noisy scattered field $\tilde{\mathbf{E}}_{\mathrm{R}}^{\text {scat }}$ measured (or synthetically created) at $\left(x_{\mathrm{R}}^{m}, y_{\mathrm{R}}^{m}\right)$, $m=1, \ldots, N_{\mathrm{R}}$. We wish to find a vector of contrast coefficients, $\mathbf{d}(k)=d_{k}, k=1, \ldots, N$, that will minimize the difference of $\tilde{\mathbf{E}}_{\mathrm{R}}^{\text {scat }}$ and $\mathbf{E}_{\mathrm{R}}^{\text {scat }}$ in the least square sense:

$$
\begin{aligned}
\mathbf{d}_{l s} & =\arg \min _{\mathbf{d}}\left\|\tilde{\mathbf{E}}_{\mathrm{R}}^{\text {scat }}-\mathbf{E}_{\mathrm{R}}^{\text {scat }}\right\|^{2}=\arg \min _{\mathbf{d}}\left\|\tilde{\mathbf{E}}_{\mathrm{R}}^{\text {scat }}-\mathbf{G}_{\mathrm{R}} \mathbf{J}\right\|^{2} \\
& =\arg \min _{\mathbf{d}}\left\|\tilde{\mathbf{E}}_{\mathrm{R}}^{\text {scat }}-j \omega \varepsilon_{0} \mathbf{G}_{\mathrm{R}} \operatorname{diag}\{\mathbf{d}\} \mathbf{E}_{\mathrm{v}}\right\|^{2}=\arg \min _{\mathbf{d}}\left\|\tilde{\mathbf{E}}_{\mathrm{R}}^{\text {scat }}-\mathbf{H d}\right\|^{2},
\end{aligned}
$$

where $\mathbf{H}=j \omega \varepsilon_{0} \mathbf{G}_{\mathrm{R}} \operatorname{diag}\left\{\mathbf{E}_{\mathrm{v}}\right\}$. It should be noted here that $\mathbf{E}_{\mathrm{v}}$ (and hence $\mathbf{H}$ ) is a function of $\mathbf{d}$ (see (5)); and (10) is a nonlinear minimization in $\mathbf{d}$. The reconstruction of d can be improved assuming that it is sparse (i.e. many of contrast coefficients $d_{k}$ $k=1, \ldots, N$, are zero) [4]. With this assumption (10) should be minimized under a sparsity promoting constraint, $\|\mathbf{d}\|_{1} \leq \gamma$. Using optimization transfer to find a surrogate function necessary to implement closed form iterations as described in $[5,6]$, the $n^{\text {th }}$ update of the iterative solution to (10) is formulated as 


$$
\begin{aligned}
\mathbf{d}^{(n+1)} & =\mathbf{d}^{(n)}+\frac{1}{\rho} \mathbf{H}^{(n) \mathrm{H}}\left(\tilde{\mathbf{E}}_{\mathrm{R}}^{\text {scat }}-\mathbf{H}^{(n)} \mathbf{d}^{(n)}\right) \\
\mathbf{d}^{(n+1)} & =T\left(\mathbf{d}^{(n+1)}\right) .
\end{aligned}
$$

Here, $T($.$) is a soft-threshold function and parameter \rho$ satisfies $\rho>s_{\max } / 2$, where $s_{\max }$ is the maximum singular value of the matrix product $\mathbf{H}^{(n)} \mathbf{H}^{(n) \mathrm{H}}$, to guarantee nonincreasing iterations $[5,6]$. It should be noted that setting $\rho$ to a very large value results in slower convergence; in other words, $\rho$ trades-off stability with fast convergence. Equation (11) is iterated in the same way as the Born iterative method inversion would be $[5,6]$ : Before the first iteration, $\mathbf{H}^{(1)}$ is computed using the Born approximation, i.e. $\mathbf{H}^{(1)}=j \omega \varepsilon_{0} \mathbf{G}_{\mathrm{R}} \operatorname{diag}\left\{\mathbf{E}_{\mathrm{v}}^{\mathrm{inc}}\right\}$. Next, $\mathbf{d}^{(2)}$ is updated using (11) assuming $\mathbf{d}^{(1)}=0$. During the second iteration, (5) is solved for $\mathbf{J}^{(2)}$ to compute $\mathbf{H}^{(1)}$; then $\mathbf{E}_{\mathrm{v}}^{(2)}$ is computed using $\mathbf{E}_{\mathrm{v}}^{(2)}(k)=\mathbf{J}^{(2)}(k) /\left(j \omega \varepsilon_{0} \mathbf{d}^{(2)}(k)\right) k=1, \ldots, N ; \quad$ and finally $\mathbf{H}^{(2)}$ is computed using $\mathbf{H}^{(2)}=j \omega \varepsilon_{0} \mathbf{G}_{\mathrm{R}} \operatorname{diag}\left\{\mathbf{E}_{\mathrm{v}}^{(2)}\right\}$. This iterative procedure is repeated until $\mathbf{d}^{(n)}$ converges. The computational cost of these iterations is dominated by the cost of solving (5) for $\mathbf{J}$. To allow for the reconstruction of electrically large scatterers, solution of (5) is accelerated using a 2D implementation of the adaptive integral method (AIM) [8]. It is noted that the notion of sparsity-regularized Born iterations can easily be extended to allow for multiple transmitters and frequencies.

\section{Numerical Results}

To demonstrate the benefits of the sparsity regularization, the proposed method is used to determine the relative permittivity profile of a scatterer using synthetically created scattered fields $\tilde{\mathbf{E}}_{\mathrm{R}}^{\text {scat }}$. The scatterer's original profile and transmitter-receiver configuration are shown in Fig. 2. The relative permittivities of the regions shown in blue, yellow, and red are 1.0, 1.5, and 2.0, respectively. The $21 \mathrm{~m} \times 21 \mathrm{~m}$ scatterer is discretized using $N=19600$ square cells with edge length $0.15 \mathrm{~m}$. It is excited at five frequencies $f=\{40,80,120,160,200\} \mathrm{MHz}$ using $N_{\mathrm{T}}=72$ line-source transmitters. The scattered fields are computed at $N_{\mathrm{R}}=72$ receiver points. All transmitters and receivers are located on a circle of radius $15.2 \mathrm{~m}$ centered at the midpoint of the scatterer. Figs. 3(a) and (b) present the images of the relative permittivity profile converged after 80 Born iterations regularized by sparsity and Tikhonov regularization that uses conjugate gradient iterations [1]. It is clear from Fig. 4 that sparsity-regularized Born iterations produce a sharper image.

\section{References}

[1] D. Colton, H. W. Engl, A. K. Louis, J. R. McLaughlin, and W. Rundell (eds), Surveys on Solution Methods for Inverse Problems, Springer, New York.

[2] Y. M. Wang and W. C. Chew "An iterative solution of two-dimensional electromagnetic problem," Int. J. Imaging Syst. Technol., vol. 1, pp. 100-108, 1989.

[3] J. A Marble and A. O. Hero, "Phase distortion correction for see-through-the-wall imaging radar," IEEE Int. Conf. Image Processing, pp. 2333-2336, 2006. 
[4] I. Daubechies, M. Defrise, and C. D. Mol, "An iterative thresholding algorithm for linear inverse problems with sparsity constraint," Comm. Pure App. Math., vol. 57, pp. 1413-1457, 2004.

[5] M. Ting, R. Raich, and A. O. Hero, "Sparse image reconstruction using sparse priors," IEEE Int. Conf. Image Processing, pp. 1261-1264, 2006.

[6] R. Raich and A. O. Hero, "Sparse image reconstruction for partially known blur functions," IEEE Int. Conf. Image Processing, pp. 637-640, 2006.

[7] A. F. Peterson, L. R. Scott, and R. Mittra, Computational Methods for Electromagnetics, Wiley-IEEE Press, Piscataway, NJ.

[8] E. Bleszynski, M. Bleszynski, and T. Jaroszewic, "AIM: Adaptive integral method for solving large-scale electromagnetic scattering and radiation problems," Radio Sci., vol. 31, pp. 1225-151, 1996

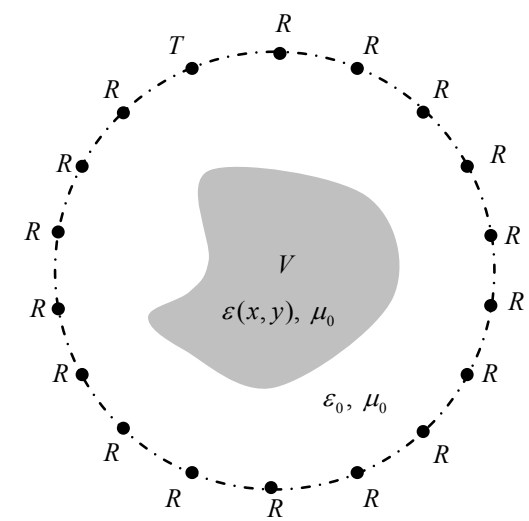

Figure 1: Description of the 2D inverse scattering problem.

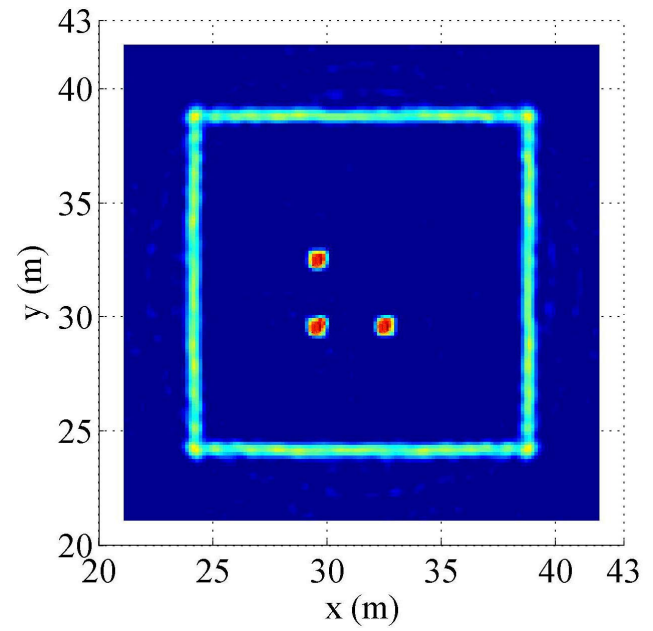

(a)

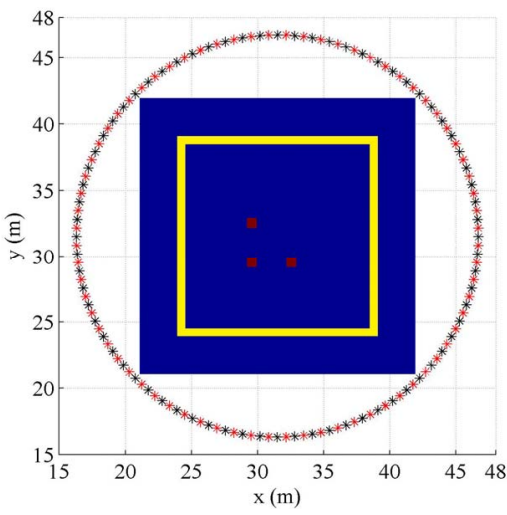

Figure 2: Original profile and transmitter-receiver configuration.

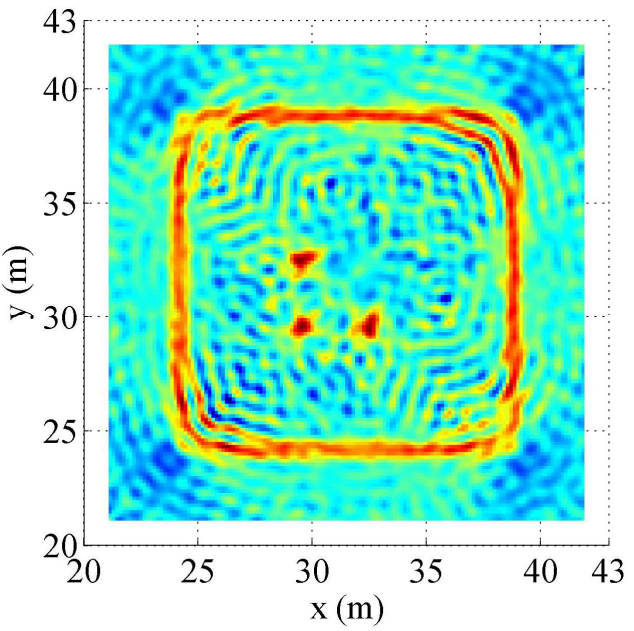

(b)

Figure 3: Images of the relative permittivity profile converged after 80 Born iterations regularized by (a) sparsity and (b) Tikhonov regularization that uses conjugate gradient iterations. 